1 Universidade Estadual do Oeste do Paraná (Unioeste) - Marechal Cândido Rondon, Paraná (PR), Brasil.

brunabratti@gmail.com

2 Universidade Estadual do Oeste do Paraná (Unioeste), Programa de Pós-Graduação de Biociências e Saúde Cascavel, Paraná (PR), Brasil.

beatriz.oliveira@unioeste.br

3 Universidade Estadual do Oeste do Paraná (Unioeste), Programa de Pós-Graduação de Biociências e Saúde Cascavel, Paraná (PR), Brasil.

clausviera@hotmail.com

4 Universidade Estadual do Oeste do Paraná (Unioeste), Centro de Ciências Biológicas e da Saúde - Cascavel, Paraná (PR), Brasil.

anatbguimaraes@gmail.com

5 Universidade Estadual do Oeste do Paraná (Unioeste) - Cascavel, Paraná (PR), Brasil. calenf3@gmail.com

\section{Avaliação da implementação da Rede Mãe Paranaense em três Regionais de Saúde do Paraná}

\author{
Evaluation of the implementation of the Rede Mãe Paranaense in \\ three Health Regions of Paraná
}

Bruna Regina Bratti Frank1, Beatriz Rosana Gonçalves de Oliveira Toso ${ }^{2}$, Cláudia Silveira Viera ${ }^{\mathbf{3}}$, Ana Tereza Bittencourt Guimarães $\mathbf{4}^{4}$ Sebastião Caldeira ${ }^{5}$

RESUMO O objetivo deste estudo foi o de avaliar a implementação da Rede Mãe Paranaense em três Regionais de Saúde (RS) do Paraná: $9^{\mathrm{a}}, 10^{\mathrm{a}}$ e $17^{\mathrm{a}}$. A pesquisa avaliou a Rede nos anos anteriores $(2010,2011)$ e posteriores $(2012,2013)$ a sua implantação. Optou-se por coleta de dados documental, com formulário validado contemplando cinco domínios, cuja média resultou no Índice Avaliativo (IA), dos quais se considerou adequados valores acima de 70 e inadequados, abaixo desse valor. A $10^{\text {a }}$ RS apresentou o melhor IA após implantação da rede, seguida da $17^{\mathrm{a}}$, e a $9^{\mathrm{a}}$ não atingiu valores adequados, mostrando necessidade de melhorar a estrutura da rede, principalmente em relação à qualificação dos profissionais quanto aos sistemas de informação.

PALAVRAS-CHAVE Avaliação de programas e projetos de saúde; Avaliação em saúde; Atenção Primária à Saúde; Saúde materno-infantil.

ABSTRACT The objective of this study was to evaluate the implementation of Rede Mãe Paranaense (Mother Network of the State of Paraná) in three Health Regions (RS) in the State of Paraná: the 9th, 10th and 17th ones. The research assessed their implementation also before (2010, 2011) as after $(2012,2013)$ it carrying out. We opted for documental data collection that applied a validated form encompassing five domains and whose average resulted in the Evaluative Index (IA). From this IA, values above the score 70 were considered adequate, while those below were considered inadequate. The 10th RS presented the best IA after the Rede deployment, followed by the 17th, while the 9th $R S$ did not reach adequate values, showing the need to improve the Rede structure, mainly concerning to the professional's qualification with regard to information systems.

KEYWORDS Program evaluation; Health evaluation; Primary Health Care; Maternal and child health. 


\section{Introdução}

As políticas públicas de saúde voltadas à população materno-infantil têm como prioridade a atenção integral às mulheres na gestação e à criança nos primeiros anos de vida, visando à garantia de assistência à saúde para ambas, além de promover estratégias para prevenção e redução da mortalidade materno-infantil. O acompanhamento em saúde a essa população é essencial para avaliar o impacto de mudanças sociais e econômicas, assim como de eventuais avanços e/ou retrocessos na disponibilidade e qualidade dos serviços de saúde (SÃo PAULO, 2011; VICTORA ET AL., 2011).

Nesse contexto, em 2012, a Secretaria de Estado da Saúde do Paraná (Sesa/PR) implantou o Programa Rede Mãe Paranaense (PRMP), fundamentado no marco conceitual das Redes de Atenção à Saúde (RAS) adotadas no Paraná como modelo de atenção para melhoria do acesso e da qualidade nas ações prestadas à população. Toda estruturação das RAS baseia-se no fortalecimento e organização da Atenção Primária à Saúde (APS) a partir da captação precoce da gestante e seu acompanhamento, e da criança até um ano de vida (MENDES, 2011; PARANÁ, 2012).

O PRMP levou em consideração a evolução dos indicadores de mortalidade materna e infantil no estado do Paraná. A Razão de Mortalidade Materna (RMM), que era de 57,64/100.000 Nascidos Vivos (NV) em 2002, aumentou para 65,11/100.000NV em 2010, e a mortalidade infantil, que era de 16,72/1.000NV em 2002, diminuiu para 12,15/1.000NV em 2010, e mantém-se próxima a esse patamar (PARANÁ, 2012).

Dos óbitos maternos registrados até 2012, $85 \%$ foram considerados evitáveis e $71 \%$ atribuídos à atenção dispensada à gestante durante o pré-natal, puerpério e assistência hospitalar. Em relação aos óbitos infantis, 60,5\% dos óbitos registrados em 2011 foram considerados evitáveis (PARANÁ, 2012).

Diante desses indicadores, a Sesa/PR estabeleceu um conjunto de ações para implantação do PRMP, quais sejam, a captação precoce da gestante, a realização de no mínimo seis consultas de pré-natal e uma de puerpério, a estratificação de risco de gestantes e crianças, o atendimento ambulatorial especializado e a vinculação das gestantes aos hospitais de referência conforme o risco. Essas ações, incluindo a análise dos indicadores de mortalidade materno-infantil, constituíram-se em pressupostos para a organização e funcionamento do PRMP (PARANÁ, 2012).

Para verificar os efeitos que a implantação de um serviço ou programa está causando na saúde do público a que se dirige, é necessária sua avaliação sistematizada. Sendo assim, este estudo teve como objetivo avaliar o processo de implementação do PRMP em três Regionais de Saúde (RS) do Paraná.

\section{Método}

Trata-se de uma pesquisa avaliativa, longitudinal, de abordagem quantitativa, realizada em três RS do Paraná, sendo a $9^{\mathrm{a}}$, de Foz do Iguaçu, a 10 , de Cascavel e a $17^{\mathrm{a}} \mathrm{RS}$, de Londrina.

Optou-se por coleta de dados documental referente aos anos anteriores (2010 e 2011) e posteriores (2012 e 2013) à implantação do PRMP, realizada a partir dos Sistemas de Informação em Saúde (SIS), a saber: Sistema de Informação de Nascidos Vivos (Sinasc), Sistema de Acompanhamento do Programa de Humanização no Pré-Natal e Nascimento (Sisprenatal), Sistema de Informação da Atenção Básica (Siab), Sistema de Informação de Mortalidade (SIM), Sistema de Informação de Imunizações (SI-PNI) e dados do Departamento de Informática do SUS (Datasus) relativos a cada município. O fato de esses dados serem secundários pode se constituir em limitação do estudo.

Para a coleta e registro dos dados, utilizou-se um instrumento validado na 
pesquisa por meio de Análise Fatorial, contendo questões que contemplam os indicadores de saúde materno-infantis propostos pela Linha Guia do PRMP, aqui chamados de domínios e apresentados no quadro 1, a seguir.

Quadro 1. Apresentação dos domínios, variáveis que os compõem e equações para sua obtenção. Cascavel, 2015

\begin{tabular}{|c|c|c|}
\hline Domínio & Variáveis & Equações \\
\hline $\begin{array}{l}\text { 1) Consultas e pre- } \\
\text { venção materna }\end{array}$ & $\begin{array}{l}\text { Número de consultas, exames do } 10 \text { trimestre, } \\
\text { exames de } 20 \text { trimestre, exames de } 30 \text { tri- } \\
\text { mestre, teste rápido de sífilis, teste rápido de } \\
\text { gravidez e teste rápido de HIV, divididas pelo } \\
\text { número de gestantes. }\end{array}$ & $\begin{array}{l}\text { Variação de } 0 \text { a } 14 \\
\text { Eq. } 1 \text { ) Consultas }=(n \text { consultas }+ \text { exames } 10 \text { tri } \\
+ \text { exames } 20 \text { tri }+ \text { exames } 30 \text { tri }+ \text { sífilis }+ \text { gravi- } \\
\text { dez }+ \text { HIV) / n gestante }\end{array}$ \\
\hline 2) Gestão & Contratualização e estratificação. & $\begin{array}{l}\text { Variação de } 0 \text { a } 4 \\
\text { Eq. 2) Gestão = Estratificação + Contratuali- } \\
\text { zação }\end{array}$ \\
\hline $\begin{array}{l}\text { 3) Seguimento da } \\
\text { Criança (Cobertura } \\
\text { vacinal) }\end{array}$ & $\begin{array}{l}\text { Hepatite B, Pentavalente, Sabin ou Salk, Ba- } \\
\text { cilus Calmette-Guérin (BCG), Tríplice Viral } \\
\text { (VTV), Febre Amarela, Rotavírus e Pneumo- } \\
\text { cóccica } 10 .\end{array}$ & $\begin{array}{l}\text { Variação de } 0 \text { a } 16 \\
\text { Eq. 3) Seguimento = Hepatite B + Pentavalen- } \\
\text { te + Sabin ou Salk + BCG + VTV + Febre Ama- } \\
\text { rela + Rotavírus + Pneumocóccica } 10\end{array}$ \\
\hline 4) Risco & Tipo de parto e mortalidade materna & $\begin{array}{l}\text { Variação de } 0 \text { a } 4 \\
\text { Eq. 4) Risco = Parto + Escore de mortalidade } \\
\text { materna }\end{array}$ \\
\hline $\begin{array}{l}\text { 5) Mortalidade } \\
\text { Infantil }\end{array}$ & $\begin{array}{l}\text { Mortalidade neonatal precoce, mortalidade } \\
\text { neonatal tardia e mortalidade pós-neonatal. }\end{array}$ & $\begin{array}{l}\text { Variação de } 0 \text { a } 6 \\
\text { Eq. 5) Mortalidade Infantil = Escore de morta- } \\
\text { lidade neonatal tardia + Escore de mortalidade } \\
\text { neonatal precoce + Escore de mortalidade } \\
\text { pós-neonatal }\end{array}$ \\
\hline
\end{tabular}

Os cálculos dos domínios resultaram em escores normalizados em escala de zero (0) a 100, após o que se realizou a média de todos os domínios para a definição do Índice Avaliativo (IA), conforme o seguinte algoritmo (equação 6):

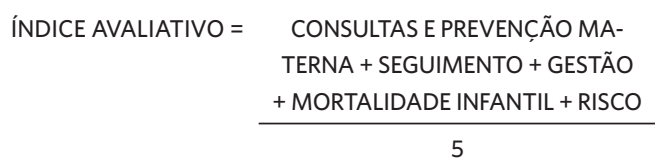

Após o cálculo do IA, os resultados foram ranqueados e classificados de acordo com os critérios de adequação do PRMP, definindo-se que valores iguais ou acima de 70 foram considerados adequados para a avaliação, e valores abaixo de 70, inadequados.
Os dados foram avaliados quanto ao padrão de distribuição por meio do teste Shapiro-Wilk, seguido da avaliação de homogeneidade das variâncias pelo teste de Cochran. As variáveis foram avaliadas pelo teste de Anova (Análise de Variância) para medidas repetidas, seguido pelo teste de acompanhamento de Fisher LSD (Least Significant Difference). Utilizou-se o nível de significância de 0,05 , sendo tais análises realizadas no programa Statistica 7.0.

A pesquisa desenvolveu-se de acordo com as normas da Resolução 446/2012 do Conselho Nacional de Saúde e o projeto foi aprovado por Comitê de Ética em Pesquisa com seres humanos sob parecer $n^{\circ}$ $544.107 / 2014$. 


\section{Resultados}

A tabela 1 apresenta os escores dos cinco domínios de avaliação dos indicadores materno-infantis, bem como o IA do PRMP nas três RS pesquisadas, ao longo do período avaliado.

Tabela 1. Média dos domínios e IA para cada ano de implementação do Programa Rede Mãe Paranaense. Cascavel, PR, 2015

\begin{tabular}{|c|c|c|c|c|}
\hline Domínio/RS & 2010 & 2011 & 2012 & 2013 \\
\hline \multicolumn{5}{|c|}{ Consultas e prevenção materna } \\
\hline 9ạ RS & 0,0 & 0,0 & 0,0 & 0,0 \\
\hline $10^{\mathrm{a}} \mathrm{RS}$ & 0,0 & 0,0 & 0,0 & 46,86 \\
\hline $17 \stackrel{\text { RS }}{ }$ & 0,0 & 7,48 & 7,14 & 49,32 \\
\hline \multicolumn{5}{|l|}{ Gestão } \\
\hline 9a RS & 33,33 & 52,78 & 55,56 & 55,56 \\
\hline $10^{\mathrm{a}} \mathrm{RS}$ & 25,00 & 100,00 & 100,00 & 100,00 \\
\hline 17 a $R S$ & 47,62 & 47,62 & 83,33 & 83,33 \\
\hline \multicolumn{5}{|c|}{ Seguimento da Criança } \\
\hline 9aㅗ RS & 67,36 & 75,69 & 77,08 & 84,72 \\
\hline $10 \cong \mathrm{RS}$ & 70,75 & 71,25 & 70,25 & 77,75 \\
\hline $17 \stackrel{\mathrm{RS}}{\mathrm{N}}$ & 68,75 & 82,74 & 75,89 & 72,02 \\
\hline \multicolumn{5}{|l|}{ Risco } \\
\hline 9a RS & 69,44 & 66,67 & 72,22 & 72,22 \\
\hline $10^{\mathrm{a}} \mathrm{RS}$ & 84,00 & 81,00 & 82,00 & 71,00 \\
\hline $17 \underline{a} \mathrm{RS}$ & 71,43 & 72,62 & 75,00 & 71,43 \\
\hline \multicolumn{5}{|c|}{ Mortalidade Infantil } \\
\hline 9 a $R S$ & 90,73 & 75,92 & 81,46 & 77,76 \\
\hline $10^{\mathrm{a}} \mathrm{RS}$ & 89,99 & 85,98 & 88,66 & 88,66 \\
\hline 17 a $R S$ & 84,91 & 84,10 & 85,70 & 90,46 \\
\hline \multicolumn{5}{|c|}{ Índice Avaliativo } \\
\hline 9a RS & 52,17 & 54,21 & 57,27 & 58,05 \\
\hline 10 a $\mathrm{RS}$ & 53,95 & 67,65 & 68,18 & 76,85 \\
\hline 17 a $R S$ & 54,54 & 58,91 & 65,41 & 73,31 \\
\hline
\end{tabular}

Fonte: Base de dados da pesquisa, 2015 
Em relação ao domínio 'Consultas e Prevenção Materna', observou-se a inexistência de registros, em 2010, nas três RS. Em 2011 e 2012, a $17^{\mathrm{a}} \mathrm{RS}$ apresentou registros insignificantes e, em 2013, aumentou na $10^{\text {a }}$ para 46,86 e na $17^{\mathrm{a}} \mathrm{RS}$, para 49,32 . Ainda assim, as três RS apresentaram escores considerados inadequados para o domínio em questão após a implantação do PRMP, retratados pela ausência de dados em todo o período.

No domínio 'Gestão', a 9 ${ }^{\mathrm{a}} \mathrm{RS}$ apresentou elevação a partir de $2011(52,78)$ quando comparada a $2010(33,33) . \mathrm{Na} 10^{\text {a }}$, esse aumento se mostrou expressivo (25,00 em 2010 para 100,00 em 2011). Na 17a , a elevação ocorreu em 2012, ano de implantação do PRMP. Os resultados das três RS nos anos posteriores à implantação do PRMP permaneceram constantes, sendo que a $10^{\mathrm{a}} \mathrm{RS}$ obteve melhor avaliação para o domínio em questão (100,00 em 2012 e 2013), seguida da $17^{\mathrm{a}}$ (83,33 em 2012 e 2014). Na $9^{\mathrm{a}} \mathrm{RS}$, a avaliação demonstrou resultados inadequados após implantação (55,56 em 2012 e 2013).

Para o domínio 'Seguimento da Criança' as médias tanto dos anos pré-implantação quanto dos anos pós-implantação do PRMP obtiveram valores muito próximos para as três RS, sendo que seus resultados foram considerados adequados. Nesse caso, a $9^{\text {a }}$ RS apresentou o melhor escore em 2013 (84,72).

Quanto ao domínio 'Risco', as médias foram adequadas no período pré e pós- implantação do PRMP, com exceção da $9^{\mathrm{a}} \mathrm{RS}$ em $2010(69,44)$ e $2011(66,67)$. A melhor avaliação foi obtida pela $10^{\mathrm{a}}$ RS em 2010 $(84,00)$. Na $17^{\mathrm{a}} \mathrm{RS}$, o maior escore foi registrado em $2012(75,00)$, apresentando queda em 2013 (71,43).

O domínio 'Mortalidade Infantil' mostrou médias consideradas adequadas nas três RS. A $9^{\mathrm{a}}$ e $10^{\mathrm{a}} \mathrm{RS}$ apresentaram valores menores em 2013 (77,76 e 88,66, respectivamente) quando comparados a 2010 (90,73 e 89,99, respectivamente). A $17^{\mathrm{a}} \mathrm{RS}$, no entanto, apresentou uma evolução gradual de 84,91, em 2010, para 90,46, em 2013.

Por fim, tem-se o resultado do IA, em que, após a implantação, mostrou que a $10^{\mathrm{a}}$ foi a RS mais bem avaliada, com escore de 76,85 em 2013 , seguida da $17^{\mathrm{a}}$, com 73,31 no mesmo ano. A avaliação do IA mostrou que os resultados pós implantação do programa, para as três RS, foram superiores aos registrados nos anos anteriores, embora a $9^{\mathrm{a}} \mathrm{RS}$ não tenha atingido valores considerados adequados.

A avaliação longitudinal da implementação do PRMP nas três RS ao longo dos quatro anos avaliados é mais bem visualizada pelas representações gráficas das análises dos cinco domínios e do IA, apresentados na figura 1, na qual se observa que os domínios que apresentaram mudanças significativas após a implantação do PRMP foram 'Consultas e Prevenção Materna' e 'Gestão', sendo que os demais apresentaram estagnação durante o processo de implementação. Ainda, expõe-se na figura 1 o IA após a implantação do PRMP, para todas as RS. 
Figura 1. Médias e intervalos de confiança dos domínios do índice avaliativo do Programa Rede Mãe Paranaense nas 9ạ 10a e 17ạ Regionais de Saúde ao longo dos anos de 2010 a 2013
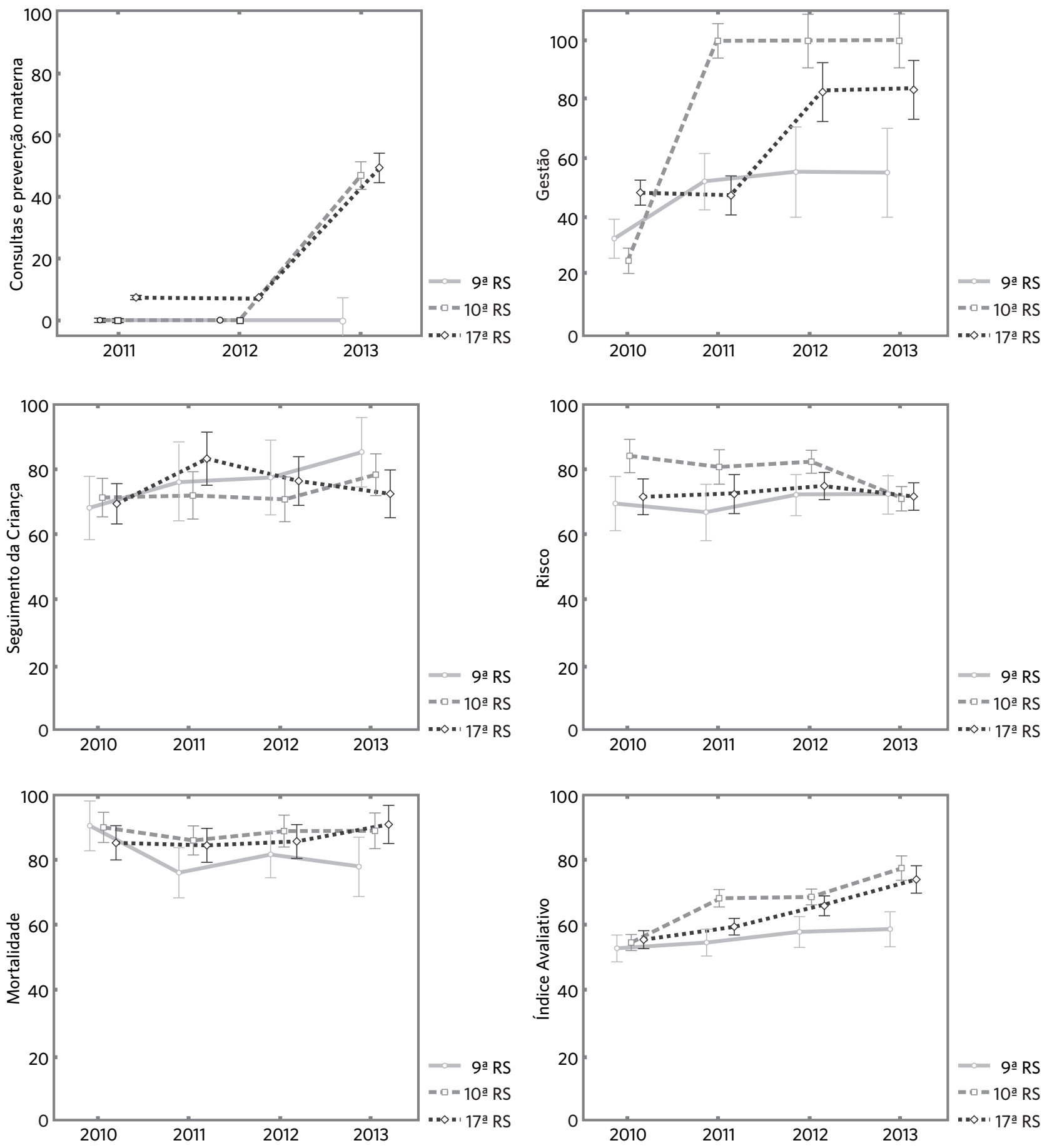


\section{Discussão}

O objetivo do acompanhamento do pré-natal é o de assegurar às gestantes o desenvolvimento saudável da gestação, permitindo a detecção e o tratamento oportuno de afecções e de fatores de risco que podem levar a complicações para a saúde materno-infantil tanto no momento do parto como no período puerperal (BRASIL, 2012).

Diante disso, o PRMP preconiza a realização de no mínimo seis consultas de pré-natal e uma de puerpério e 17 exames laboratoriais ao longo dos três trimestres gestacionais, incluindo o teste rápido de gravidez e a ultrassonografia obstétrica. Ainda, os testes rápidos para detecção do vírus HIV tipo $1 \mathrm{e}$ 2 e sífilis são imprescindíveis no $1^{\circ}$ trimestre gestacional. É por esse motivo que a detecção precoce das gestantes e sua vinculação e acompanhamento na APS é uma das principais metas do PRMP (PARANÁ, 2012).

No entanto, os resultados apresentados pelo domínio 'Consultas e Prevenção Materna' contrariam esse objetivo, evidenciando uma considerável falha nos registros de informação do Sisprenatal. Essa falha pode ser identificada principalmente em 2010, em que não há registro de informação nas três RS. Na $9^{\text {a }}$ RS, tal problema é evidenciado nos quatro anos estudados.

O Sisprenatal é um sistema eletrônico criado pelo Datasus para a coleta de informações sobre o acompanhamento de pré-natal das gestantes no contexto do Programa de Humanização do Pré-natal e Nascimento (PHPN) (ANDREUCCI; CECATTI, 2011). O programa disponibiliza instrumentos de coleta, devendo as informações ser digitadas no sistema. Qualquer falha no registro e transcrição dos dados para o sistema pode resultar em informações incompletas ou indisponíveis, comprometendo a avaliação da qualidade da assistência prestada à gestante, fato constatado em estudo realizado para avaliar a qualidade do Sisprenatal, em que as informações registradas manualmente no cartão do pré-natal propiciam melhor notificação que o sistema (ANDREUCCI ET AL., 2011).

Visando à qualidade da assistência materno-infantil, uma das ações do PRMP é a detecção e prevenção de fatores de risco durante a gestação por meio da estratificação de risco da gestante e criança, de acordo com a seguinte classificação: Risco Habitual, Intermediário e Alto Risco, sendo que o risco Intermediário foi uma ação inovada pelo PRMP. Essa estratificação permite o acompanhamento e vinculação das gestantes e crianças aos hospitais e ambulatórios conforme o risco. É válido lembrar que, dentro da rede, a gestante terá seu seguimento nos serviços de APS dos municípios e ambulatórios especializados para atenção à gestante de risco durante o pré-natal, e seu parto será realizado nos hospitais contratados com 0 PRMP (PARANÁ, 2012).

A relação entre a estratificação de risco e a contratualização dos hospitais com o PRMP, demonstrada pelo domínio 'Gestão', constitui-se em uma importante ferramenta para o fluxo da rede e possibilita a resolutividade das condições de saúde das gestantes e crianças no interior do sistema (MENDES, 2011).

Ainda que sua implantação tenha ocorrido no ano de 2012, em 2011 se iniciaram as etapas preparatórias do PRMP. Até então, gestantes e crianças eram classificadas como Baixo e Alto Risco e encaminhadas aos hospitais de referência existentes nas sedes das RS. Com a implantação do PRMP, adotou-se a estratificação de risco Intermediário e as gestantes e crianças foram vinculadas aos hospitais que possuíssem Termo de Contrato com o PRMP (PARANÁ, 2012), o que explica o aumento expressivo das médias em 2012 quando comparadas a 2010, como no caso das $9^{\mathrm{a}}$ e $10^{\mathrm{a}} \mathrm{RS}$.

Os baixos valores demonstrados para a $9^{\text {a }}$ RS após a implantação podem ser explicados, porque nem todos os municípios realizam referência de gestantes e crianças para as três classificações de risco. Em alguns casos, os riscos Habitual e Intermediário, 
por exemplo, são acompanhados no município por hospitais não contratualizados ou pertencentes à outra RS.

Em relação à contratualização dos hospitais nas RS do estudo, tem-se: na 9a ${ }^{\text {a }}$ um hospital de referência para risco Intermediário, sendo que o mesmo é referencia para Alto Risco; na $10^{\mathrm{a}}$, um hospital para risco Intermediário e um para Alto Risco; e, na $17^{\mathrm{a}}$, quatro hospitais para Intermediário e dois para Alto Risco (PARANÁ, 2012).

Tendo em vista a redução da morbimortalidade materno-infantil, faz-se necessário identificar as situações de risco gestacional o mais precocemente possível. Nesse sentido, o acolhimento às gestantes com classificação de risco pressupõe agilidade no atendimento e definição da necessidade de cuidado e densidade tecnológica, ofertados a essa população (BRASIL, 2012).

No que diz respeito ao acompanhamento da criança, o domínio 'Seguimento' demonstra dados referentes à imunização. Formulado em 1973 e institucionalizado em 1975, o PNI foi criado tendo como meta operacional básica o alcance de $100 \%$ da cobertura vacinal para menores de um ano (BRASIL, 2003). Entretanto, os resultados apresentados, mesmo com escores considerados adequados, evidenciam uma cobertura abaixo do preconizado nas três RS.

A vacinação infantil contribui para a prevenção de doenças infectocontagiosas, controle de surtos epidêmicos e erradicação de doenças, como é o caso da poliomielite e varíola. Sendo assim, a imunização no primeiro ano de vida constitui-se em um importante indicador no controle da mortalidade infantil (PUGLIESI; TURA; ANDREAZZI, 2010; SOUZA; VIGO; PALMEIRA, 2012).

Contudo, apenas a segurança e eficácia dos imunobiológicos não são suficientes para a garantia de sucesso dos programas de imunização. É preciso que os profissionais os operacionalizem conforme as recomendações exigidas, observando a conservação, manejo, técnica de administração, registro nos SIS, busca ativa de faltosos e suscetíveis e acompanhamento pós-vacinal, e, também, que a população tenha conhecimento de sua importância para redução da morbimortalidade infantil em todo País. Em relação aos profissionais, é necessária a supervisão, monitoramento e avaliação das salas de vacina (VASCONCELOS; ROCHA; AYRES, 2012) na APS para que se possa alcançar a cobertura vacinal ideal, conforme estimado. No caso da população, torna-se imprescindível trabalhar com educação em saúde nas unidades de APS, tendo em vista a ampliação do entendimento dessa prática de saúde e seus benefícios, enfatizando a manutenção do esquema vacinal e estimulando a promoção da saúde (SANTOS ET AL., 2011).

Quanto à assistência em saúde às gestantes, tem-se o domínio 'Risco', que expressa tanto o tipo de parto realizado como a RMM das três RS. Essa relação é diretamente proporcional ao tipo de parto, em que quanto maior a incidência de parto cesáreo, maior o risco de mortalidade materna (MACHADO JUNIOR ET AL., 2009).

A Organização Mundial da Saúde (OMS) preconiza que o total de partos cesáreos realizados em um serviço de saúde varie de $10 \%$ a $15 \%$, visto que é a porcentagem que apresenta indicação precisa de cesariana, ou seja, existe uma situação real em que, para a preservação da saúde materno-infantil, indica-se que o parto seja realizado cirurgicamente (OMS, 2015).

Nesse sentido, esta pesquisa demonstrou oscilações nos escores para as três RS, sendo que a $10^{\mathrm{a}}$ obteve melhores resultados nos anos pré-implantação do PRMP quando comparada aos anos subsequentes. A $17^{\mathrm{a}}$ apresentou a melhor avaliação em 2012 e a $9^{a} \mathrm{RS}$ apresentou os melhores resultados em 2013, após a implantação.

Em 2010, 58,52\% dos partos realizados no Paraná ocorreram por cesárea; em 2013, a porcentagem foi de $63,55 \%$. Essa estatística é similar aos dados encontrados para a $10^{\mathrm{a}} \mathrm{RS}$, em que o registro de partos cesáreos subiu 
de $57,56 \%$ em 2010 para $62,22 \%$ em 2013 . Na $9^{\text {a }} \mathrm{RS}$, os partos cesáreos subiram de $54,34 \%$ em 2010 para $59,63 \%$ em 2013 , e na $17^{\mathrm{a}}$, em contrapartida, os registros foram de $63,55 \%$ em 2010 e de $42,58 \%$ em 2013, reduzindo em $20 \%$ o número de cesáreas registradas. Visto que o incentivo ao parto natural é uma das metas propostas pelo PRMP e constitui-se em importante indicador para a avaliação da Rede, essa ainda é uma meta a ser atingida (PARANÁ, 2012).

A cesárea, quando realizada sob indicações médicas específicas, é um procedimento indispensável para a saúde materno-infantil (HOLANDA ET AL., 2011), porém sua utilização exige um conhecimento profundo dos riscos e implicações existentes. Assim sendo, torna-se imprescindível a qualificação na atenção à gestante para garantir que a decisão pela via de parto considere os ganhos em saúde e seus possíveis riscos de forma claramente informada pelos profissionais de saúde, visando à redução da mortalidade materno-infantil (BRASIL, 2015).

A redução da RMM é uma preocupação mundial e consta como a $5^{\text {a }}$ meta dos Objetivos de Desenvolvimento do Milênio (ODM) das Nações Unidas do ano de 2000, assinado por 191 países que se comprometeram a reduzir em $75 \%$ a RMM entre 2000 e 2015. No Brasil, a RMM decresceu de 143/100.000NV em 1990 para 70/100.000NV em 2010, sendo que em 2011 a taxa foi de 64/100.000NV (PNUD, 2012).

Quanto à RMM no Paraná, o índice de óbitos maternos decresceu de 65,11/100.000NV em 2010 para 39,28/100.000NV em 2013 (PARANÁ, 2012). Esse declínio significativo da RMM no Paraná nos últimos anos ficou também evidenciado na $9^{\mathrm{a}}$ RS, cujo valor de 64,73/100.000NV em 2010 diminuiu para $31,26 / 100.00 \mathrm{NV}$ em 2013; na $17^{\mathrm{a}}$, reduziu de 59,97/100.000NV em 2010 para 40,96/100.000NV em 2013. No entanto, na $10^{\text {a }} \mathrm{RS}$, os dados apresentados são piores do que a estatística do Estado, sendo que a RMM aumentou de 54,13/100.000NV em
2010 para $66,27 / 100.000 \mathrm{NV}$ em 2013 , confirmando a diminuição dos escores apresentados no domínio 'Risco'.

Mesmo revelando sinais de progresso, a mortalidade materna, tanto no Brasil como no Paraná, ainda apresenta resultados muito aquém do esperado para atingir a meta do ODM, que consiste em reduzir a RMM em três quartos até 2015. Nesse sentido, ações que contribuam para a redução da RMM são imprescindíveis, tais como a melhoria no acesso e qualidade dos serviços e ações de saúde, contribuindo para a identificação precoce de fatores de risco gestacionais, a continuidade das ações em saúde, uma melhor integração entre os níveis assistenciais e profissionais e, principalmente, o fortalecimento do vínculo das mulheres com os serviços e equipes de saúde (MANDU; ANTIQUEIRA; LANZA, 2009).

No que se refere à saúde das crianças, tem-se o domínio 'Mortalidade Infantil', representado pelos escores de mortalidade neonatal precoce, tardia e pós-neonatal. A pesquisa demonstrou consideráveis oscilações nas médias apresentadas pela $9^{\mathrm{a}} \mathrm{e}$ $10^{\mathrm{a}} \mathrm{RS}$, sendo que os escores de 2010 foram superiores aos de 2013, pós-implantação do PRMP. A $17^{\text {a }}$ foi a RS que obteve maior impacto da implantação do PRMP, apresentando um aumento gradual no decorrer dos quatro anos, sendo que em 2013 apresentou o maior escore avaliado.

O Coeficiente de Mortalidade Infantil (CMI) no Brasil decresceu de 26,04/1.000NV em 2002 para 17,22/1.000NV em 2010. Em 2011, o Brasil atingiu a meta estabelecida pelo ODM, com um CMI de 15,3/1.000NV, sendo a meta para 2015 de $15,7 / 1.000 \mathrm{NV}$ (PNUD, 2012)

No Paraná, a redução foi lenta e desigual, sendo que em 2010 o coeficiente era de 12,15/1.000NV e decresceu para 10,94/1.000NV em 2013 (PARANÁ, 2012). Em contrapartida, os resultados apresentados pelas $9^{\text {a }}$ e $10^{\text {a }}$ RS contrariam os dados nacionais e do Estado, sendo que o CMI na $9^{\text {a }}$ cresceu de 
16,66/1.000NV em 2010 para 22,67/1.000NV em 2013 , e o da $10^{\text {a }}$, de $11,36 / 1.000 \mathrm{NV}$ em 2010 para $18,02 / 1.000 \mathrm{NV}$ em 2013. A $17^{\mathrm{a}}$ RS obteve os melhores resultados, apresentando um decréscimo de 11,65/1.000NV em 2010 para 9,58/1.000NV em 2013.

A redução da mortalidade infantil ainda se constitui um desafio para os serviços de saúde (BRASIL, 2009), evidenciado pela estagnação do Paraná nos últimos anos e pelos resultados negativos das $9^{\mathrm{a}}$ e $10^{\mathrm{a}} \mathrm{RS}$ nos anos avaliados.

As principais causas de mortalidade infantil estão relacionadas ao acesso e utilização dos serviços de saúde e à qualidade da assistência prestada no pré-natal, no parto e ao recém-nascido. Estatísticas demonstram que as afecções perinatais se mantêm como a primeira grande causa de óbito em menores de um ano no Brasil (BRASIL, 2009).

Nesse sentido, dentre os óbitos infantis, os óbitos neonatais precoces têm sido recomendados como um dos indicadores mais apropriados à análise da assistência obstétrica e neonatal e de utilização dos serviços de saúde, permitindo maior visibilidade do problema e propiciando a identificação de ações de prevenção de óbitos neonatais precoces evitáveis (BRASIL, 2009).

Sobre esse aspecto, $60,5 \%$ dos óbitos infantis registrados em 2011 foram considerados evitáveis, ou seja, diante da garantia de acesso em tempo oportuno aos serviços qualificados de saúde, poderiam ter sido evitados. As intervenções dirigidas à redução da mortalidade infantil dependem de mudanças estruturais relacionadas às condições de vida da população e de ações diretas definidas pelas políticas públicas de saúde (BRASIL, 2009).

A avaliação do impacto da implantação do PRMP nas três RS em estudo pode ser observada pela apresentação dos resultados do IA. Os domínios que mais influenciaram nesse resultado foram 'Consultas e Prevenção Materna', devido à inexistência de dados que geraram resultados consideravelmente abaixo do preconizado para a avaliação, e 'Gestão', que contribuiu para os resultados positivos após a implantação do PRMP, principalmente para a $10^{\mathrm{a}} \mathrm{RS}$. Os demais domínios, apesar de terem contribuído positivamente para a avaliação do IA, não demonstraram variações significativas.

Assim, é possível verificar a importância de avaliar o processo de implantação de programas, visto que essa avaliação é fundamental para entender os motivos pelos quais a intervenção está ou não sendo efetivada e o que fazer para melhorá-la. As ações de avaliação são consideradas essenciais para o planejamento e gestão e funcionam como instrumento de suporte para a formulação de políticas, para o processo de tomada de decisão e para a formação dos sujeitos envolvidos. Na gestão em saúde, a avaliação de políticas deve ter como finalidade a melhoria dos serviços e ações, a prestação de contas e a produção de conhecimentos considerados úteis para atingir a qualidade na prática em saúde (CRUZ, 2011), principalmente no que diz respeito à atenção à saúde das mulheres e crianças no âmbito da APS.

Consoante essa concepção e visando ao fortalecimento da APS em todo o estado, a Sesa/PR propôs o Programa de Qualificação da APS (APSUS) com o objetivo de aprimorar o processo de trabalho dos profissionais de saúde por meio de oficinas de educação permanente, as quais propõem, dentre outras metas, a qualificação dos profissionais da APS que atuam no seguimento materno-infantil como forma de promover a melhoria dos indicadores de saúde em todo o estado (PARANÁ, 2012).

\section{Considerações finais}

Os domínios 'Gestão' e 'Consultas e Prevenção Materna' apresentaram maior influência da implantação do PRMP, ainda que os escores deste último domínio tenham permanecido abaixo do preconizado. Os demais 
domínios mostraram oscilações pouco significativas e os escores após a implantação do PRMP foram considerados adequados nas três RS. A RS que apresentou o melhor IA após a implantação do PRMP foi a $10^{\mathrm{a}}$, seguida da $17^{\mathrm{a}}$, sendo que os valores apresentados no ano de 2013 atingiram escores considerados adequados. Por outro lado, a $9^{\text {a }}$ RS, além de apresentar médias que oscilaram pouco ao longo dos quatro anos, não atingiu os resultados esperados pela avaliação.

Com a avaliação realizada, levantaram-se duas importantes considerações quanto à estruturação do PRMP. A primeira é em relação a alimentação e atualização dos SIS, uma vez que se constituem como as principais ferramentas de informação para avaliação da qualidade da assistência prestada à população e fornecem subsídios para implantação, acompanhamento e avaliação dos modelos de atenção à saúde. Um dos maiores problemas para a análise de informações em saúde é a confiabilidade dos indicadores gerados, razão pela qual sugere-se maior monitoramento na coleta e processamento dos dados dos sistemas para a correção de falhas e aperfeiçoamento dos dados produzidos.

A segunda, os resultados possibilitaram uma crítica ao cumprimento das ações preconizadas pelo PRMP, uma vez que os dados observados nas $9^{\mathrm{a}}$ e $10^{\mathrm{a}} \mathrm{RS}$, no que diz respeito às taxas de mortalidade materna $\mathrm{e}$ infantil e à incidência de partos cesáreos, são inferiores às metas propostas. Nesse sentido, é necessário avaliar a qualidade da atenção prestada às gestantes no pré-natal para identificar as situações de risco e vulnerabilidade que exigem maior complexidade de ações e cuidado, desenvolvendo a importância de se evitar óbitos materno-infantis e a realização de partos cesáreos em número elevado.

A pesquisa mostrou que a implantação do PRMP, mesmo com as falhas identificadas, tem apresentado impacto positivo nos indicadores de saúde materno-infantil. Seu processo de implementação é incipiente e ainda há muito a melhorar quanto à forma em que se encontra estruturado e como tem sido implementado, principalmente no que diz respeito à qualificação dos profissionais que permeiam esse processo, para que seja possível promover a atenção à saúde das mulheres e crianças de forma eficiente e resolutiva, permitindo, assim, a redução da mortalidade materna e infantil no Paraná.

\section{Referências}

\footnotetext{
ANDREUCCI, C. B.; CECATTI, J. G. Desempenho de indicadores de processo do Programa de Humanização do Pré-natal e Nascimento no Brasil: uma revisão sistemática. Cad. Saude Publica, Rio de Janeiro, v. 27, n. 6, p. 1053-1064, jun. 2011.
}

ANDREUCCI, C. B. et al. Sisprenatal como instrumento de avaliação de qualidade da assistência à gestante. Rev Saúde Pública, São Paulo, v. 45, n. 5, p. 854-63, 2011.

BRASIL. Ministério da Saúde. Secretaria de Atenção à Saúde. Atenção ao pré-natal de baixo risco. Brasília, DF: Ministério da Saúde, 2012. 
Ministério da Saúde. Secretaria de Ciência, Tecnologia e Insumos Estratégicos. Comissão Nacional de Incorporação de Tecnologias no SUS (CONITEC). Diretrizes de Atenção à Gestante: a operação Cesariana. Brasília, DF: Ministério da Saúde, 2015.

Ministério da Saúde. Secretaria de Vigilância em Saúde. Manual de vigilância de óbito infantil e fetal e do Comitê de Prevenção do Óbito Infantil e Fetal. 2. ed. Brasília, DF: Ministério da Saúde, 2009.

Ministério da Saúde. Secretaria de Vigilância em Saúde. Programa Nacional de Imunizações - 30 anos. Brasília, DF: Ministério da Saúde, 2003.

CRUZ, M. M. Avaliação de Políticas e Programas de saúde: contribuições para o debate. In: MATTOS, R. A.; BAPTISTA, T. W. F. Caminhos para análise das politicas de saúde, [Internet], 2011. p. 181-199. Disponível em: <http://www.ims.uerj.br/ccaps/wp-content/ uploads/2011/10/Caminhos-para-An\%C3\%Allises-dePol\%C3\%ADticas-de-Sa\%C3\%BAde.pdf $>$. Acesso em: 11 maio 2015.

HOLANDA, V. R. et al. Avaliação da assistência obstétrica no Estado da Paraíba à luz das normas sanitárias. Saúde debate, Rio de Janeiro, v. 35, n. 91, p. 624-33, out./dez. 2011.

MACHADO JUNIOR, L. C. et al. Associação entre via de parto e complicações maternas em hospital público da Grande São Paulo, Brasil. Cad Saúde Pública, Rio de Janeiro, v. 25, n. 1, p. 124-32, jan. 2009.

MANDU, E. N. T.; ANTIQUEIRA, V. M. A.; LANZA, R. A. C. Mortalidade materna: implicações para o Programa Saúde da Família. Rev Enferm UERJ, Rio de Janeiro, v. 17, n. 2, p. 278-84, abr./jun. 2009.

MENDES, E. V. As redes de atenção à saúde. Brasília, DF: Organização Pan-Americana da Saúde, 2011.

ORGANIZAÇÃO MUNDIAL DE SAÚDE (OMS). Declaração da OMS sobre taxas de cesáreas, 2015.
PARANÁ. Secretaria de Estado da Saúde (SESA). Linha guia rede mãe paranaense. Paraná, 2012.

PROGRAMA DAS NAÇÕES UNIDAS PARA O DESENVOLVIMENTO (PNUD). Objetivos de Desenvolvimento do Milênio, 2012.

PUGLIESI, M. V.; TURA, L. F. R., ANDREAZZI,

F. S. Mães e vacinação das crianças: estudo de representações sociais em serviço público de saúde. Rev Bras Saude Mater Infant, Recife, v. 10, n. 1, jan./mar. 2010

SANTOS, L. B. et al.; Percepção das mães quanto à importância da imunização infantil. Rev Rene, Fortaleza, v. 12, n. 3, p. 621-626, jul./set. 2011

SÃO PAULO. Secretaria Municipal da Saúde. Saúde materno-infantil: uso de serviços de saúde para morbidade de 15 dias. São Paulo, 2011. (Boletins ISA Capital 2008, 6).

SOUZA, C. J.; VIGO, Z. L.; PALMEIRA, C. S.

Compreensão dos pais acerca da importância da vacinação infantil. Revista Enfermagem Contemporânea, Salvador, v. 1, n. 1, p. 44-58, dez. 2012.

VASCONCELOS, K. C. E.; ROCHA, S. A.; AYRES, J. A. Avaliação normativa das salas de vacinas na rede pública de saúde do Município de Marília, estado de São Paulo, Brasil, 2008-2009. Rev. Epidemiol. Serv. Saúde, Brasília, v. 21, n. 1, mar. 2012.

VICTORA, C. G. et al. Saúde de mães e crianças no Brasil: progressos e desafios. The Lancet, Londres, v. 377, n. 9780, p. 1863-1876, 2011.

\footnotetext{
Recebido para publicação em novembro de 2015 Versão final em abril de 2016 Conflito de interesses: inexistente Suporte financeiro: Conselho Nacional de Desenvolvimento Científico e Tecnológico (CNPq), Edital Universal 14/2013. processo $n \div$ 474768/2013-9
} 